
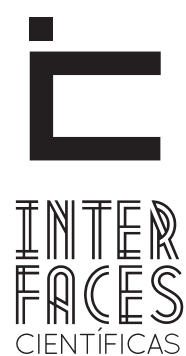

EDUCAÇÃO

\title{
PENSAR, FAZER E COMPARTILHAR: A CONSTRUÇ̄̃O COOPERATIVA ENTRE ALUNO E PROFESSOR NO FACEBOOK
}

\section{RESUMO}

Edilberto Marcelino da Gama Neto ${ }^{1}$

Valmir Alves Teixeira Júnior ${ }^{3}$

Constantes mudanças repentinas fazem parte do cotidiano da população mundial. Elas surgem de forma tão rápida e contínua que a maioria das pessoas não percebe a sua existência. Os avanços tecnológicos e as descobertas para tornar a vida mundana cada vez mais simples em meio à tempestade informacional intensa, proporcionada pela quebra de fronteiras materializada pela intermitente evolução da internet, torna o homem um ser complexo, coberto por ferramentas que o informam e intermediam a sua relação com o mundo. A forma com que uma pessoa entra em contato com as notícias globais vão além do ponto de vista apresentado pela emissora de televisão que domina a audiência de sua região, elas perpassam pela visão de correspondentes de várias partes do mundo e pelas opiniões dos personagens envolvidos no acontecimento com apenas um clique. Isso propicia a formação da opinião individual, seu compartilhamento e discussão, por meio dos sistemas comunicacionais disponíveis na rede. Este ser promotor de opiniões é o aluno contemporâneo, o ator principal deste artigo que objetiva expor a experiência efetuada em sala de aula ao tornar um grupo
Cristiane de Magalhães Porto ${ }^{3}$ Lucas Cerqueira do Vale ${ }^{4}$

do Facebook uma extensão do conteúdo trabalhado intramuros da universidade. Inicialmente, este escrito discorre a relação entre a população e o Facebook, em seguida trata da potencialidade do sistema e da escolha deste como extensão da sala de aula. Finalmente, descreve a experiência de tornar uma rede social como elo que liga a aula ao estudante. A conclusão destaca e avalia os resultados obtidos nessa primeira descrição sobre o assunto em tela. Quanto à natureza, a pesquisa se constituirá como um resumo de assunto, mas vai se debruçar sobre o tema a partir de uma perspectiva original. Do ponto de vista dos objetivos, pode-se classificá-la como uma pesquisa exploratória e descritiva. No que se refere aos procedimentos e ao objeto, se caracterizará como uma pesquisa de campo e de bibliografia. Para a realização da coleta de dados, durante a pesquisa, será utilizada, como principal instrumento, a observação direta sistemática.

\section{PALAVRAS-CHAVE}

Aluno Contemporâneo. Facebook. Experiência. 


\section{ABSTRACT}

Constant sudden changes are part of everyday life of the population. They come so fast and continuously that most people do not notice their existence. Technological advances and discoveries to make worldly life increasingly simple informational intense the storm, provided by breaking boundaries materialized by intermittent evolution of the Internet, makes man a complex being covered by tools that inform and mediate the their relationship with the world. The way a person comes into contact with the global news beyond the point of view presented by television station that dominates the audience in your area, they pervade the vision of correspondents from around the world and the opinions of the characters involved in event with just one click. This promotes the formation of individual opinion, your sharing and discussion through the communication systems available on the net. This being a promoter of the student opinions is contemporary, the main actor of this article aims to explain the experience made in the classroom to make a Facebook group an extension of the content worked intramural university. Initially this writing discusses the relationship between population and Facebook, then comes the capability of the system and the choice of this as an extension of the classroom. Finally, it describes the experience of becoming a social network as a link that connects the classroom to the student. The conclusion highlights and evaluates the results obtained in this first report on the subject at hand. The nature, the research will constitute a summary of the subject, but will not dwell on the topic from a unique perspective. From the point of view of the objectives, you can classify it as an exploratory and descriptive. Regarding the procedures and the object will be characterized as a field research and bibliography. To perform the data collection during the research, will be used as the main instrument, the systematic direct observation.

\section{KEYWORD}

Contemporary Student. Facebook. Experience.

\section{RESUMEN}

Cambios repentinos constantes son parte de la vida cotidiana de la población. Vienen tan rápido y de forma continua que la mayoría de las personas no se dan cuenta de su existencia. Avances y descubrimientos tecnológicos para hacer la vida mundana cada vez más sencilla de información intensa de la tormenta , siempre rompiendo límites materializados por la evolución intermitente de la Internet, hace del hombre un complejo está cubierto por herramientas que informary mediar el su relación con el mundo. La forma en que una persona entra en contacto con las noticias mundiales más allá del punto de vista presentado por la estación de televisión que domina a la audiencia en su área, que impregnan la visión de los corresponsales de todo el mundo y las opiniones de los personajes involucrados en evento con un solo clic. Esto promueve la formación de la opinión individual, el intercambio y la discusión através de los sistemas de comunicación disponibles en la red. Este es un promotor de las opiniones de los estudiantes es contemporánea, el actor principal de este artículo trata de explicar la experiencia adquirida en el aula para hacer un grupo en Facebook una extensión del contenido trabajó universitaria intramural . Inicialmente este escrito se analiza la relación entre la población y Facebook, luego viene la capacidad del sistema y la elección de este como una extensión del salón de clases. Por último, se describe la experiencia de convertirse en una red social como un enlace que conecta el salón de clases para los estudiantes. La conclusión destaca y evalúa los resultados obtenidos en este primer informe sobre el tema en cuestión. La naturaleza, la investigación constituye un resumen del tema, pero no voy a detenerme en el tema desde una perspectiva única. Desde el punto de vista de los objetivos, se puede clasificar como un estudio exploratorio y descriptivo. En cuanto a los procedimientos y el objeto se caracteriza como una investigación de campo y la bibliografía. Para llevar a cabo la recogida de datos durante la investigación, se utilizará como el instrumento principal , la observación directa sistemática . 


\section{PALABRAS CLAVE}

Estudiante. Contemporáneo. Facebook. Experiencia .

\section{INTRODUCÇÃO}

Estamos na era dos "Millennials", ou "Me Me Me Generation", como aborda a capa da revista americana Time (9 de maio de 2013), das discussões sobre as horas passadas online e a possibilidade do emburrecimento pela quase anulação de uma vida offline. A população mundial se rendeu ao mundo tecnológico, tornando-se conectada e dependente de uma vasta gama de recursos que foram vendidos como facilitadores e otimizadores para as atividades. Estas fazem parte do dia-a-dia da maioria dos indivíduos, como e-mail, blogs, sites de notícias, gadgets, aplicativos e as populares redes sociais, aderidas em massa pela juventude mundial.

Segundo Pedro Burgos (2013, online), o tempo gasto na vida online vem causando, desde meados de 2008 (período da consolidação do Facebook, Twitter e Youtube), algumas discussões e correntes contra a chamada hiperconexão ininterrupta, que vão da hipótese sobre a diferença do funcionamento do cérebro das pessoas que passam tempo em demasia na internet, descrita no livro Geração Superficial do Nicholas Carr (2010), passando pela "iDisorder", de Larry Rosen (2012), que defende que a obsessão por gadgets causa transtornos psiquiátricos na população mundial, e chegando aos questionamentos sobre o que acontece com as relações pessoais após o advento das redes sociais, levantados por Sherry Turkle (2011).

Todo o movimento contra a vida conectada gerou o antimovimento exposto na matéria do Alexandre Soares para a revista VIP, de abril de 2013, que defende o ambiente virtual colocando-o no patamar de fonte livre para o conhecimento. Em sua teoria, Soares
(2013, online) argumenta que todos são ignorantes, pois, a necessidade de conhecimento é geral, e como os cérebros não possuem um limite de capacidade. A internet proporciona informações sobre múltiplos assuntos e que cabe ao usuário acessar o que lhe é de interesse, independente se é classificado como fútil ou necessário pela grande massa, quanto mais tempo se navega, mais informação o usuário recebe.

Em meio a esse panorama, Paul Muller, repórter do "The Verge", experimentou passar um ano longe da internet, de 2012 até 2013, e chegou à conclusão de que os recursos online oferecidos facilitam a vida, tornam o ser humano mais produtivo, além de ser uma fuga do tédio. Em seu artigo para o "The Verge" em maio de 2013, Muller relata sua experiência e afirma que é possível viver desconectado, porém a facilidade de interação e de acesso à informação é facilitada no mundo virtual.

O contato com a internet vem atingindo de forma contínua e acelerada a parcela mais nova da população mundial, condicionando-os a uma vida conectada, que não é separada da chamada vida real. Os jovens curtem e compartilham as suas rotinas, ambições, causas e experiências, em tempo real, montando um cenário virtual onde todos estão inseridos em suas vidas, desde que aceitos em sua rede social, criando um mundo paralelo e controlado, onde passam a maior parte do seu tempo. Para atingir a essa parcela de humanos, é necessário estar conectado e oferecer-lhes experiências online como complemento da offline e vice-versa. 
Se os jovens passam tanto tempo no Facebook, porque não estender a aula utilizando-se da plataforma, incentivando-os cada vez mais a explorar em seu mundo os conhecimentos necessários para a sua vida offline Com esse cenário em mãos, foi feita uma pequena experiência no Curso de Design Gráfico da Universidade Tiradentes com o objetivo de tornar as aulas parte da vida virtual de seus alunos, utilizando um grupo fechado no Facebook como uma sala de aula que não tem hora para começar e nem para terminar.

Inicialmente este tratará da relação do mundo contemporâneo com as novas tecnologias, focando na importância das redes sociais para a população mundial e traçando um breve perfil do jovem atual. Em seguida inicia-se a descrição da experiência da utilização do Facebook como complemento da sala de aula numa turma do Curso de Design Gráfico da Universidade Tiradentes, expondo a forma como foi mesclada a aula presencial e o uso da rede social. E, finalmente, trata na conclusão dos benefícios da utilização de um recurso presente no cotidiano dos alunos para melhorar a dinâmica do ensino-aprendizagem em sala de aula.

Pretende-se assim, compartilhar a experiência e o aprendizado, além de fomentar a discussão sobre a inserção de recursos disponíveis que fazem parte do universo dos alunos na metodologia aplicada em aula, a fim de tornar a transmissão de conteúdo mais dinâmico e conferir ao aluno a oportunidade de tornar-se mais participativo no ato de construção do conhecimento.

\section{UMA REDE SOCIAL, UMA EXTENSÃO DO MUNDO}

A necessidade de se comunicar induziu o homem a descobrir novos meios para apresentar aquilo que deseja transmitir, tornando mais eficaz o processo de compreensão da sua mensagem. Percebe-se então, que a evolução da comunicação acompanha a da humanidade, já que, à medida que a sociedade muda, as formas de comunicação se adaptam as suas novas necessidades, do mesmo modo que, quanto mais moderno o sistema de comunicação se torna, maior a influência no comportamento social das pessoas. Fato comprovado ao observar a população atual e sua relação com a Rede Mundial de Computadores.

A internet tem estado cada vez mais presente na vida e no cotidiano da sociedade. Existe uma crescente aderência ao mundo virtual, levando em consideração o crescimento da rede nos últimos anos e a velocidade com que esta mudança tem acontecido.

A internet tem a missão de interligar pessoas dis- tantes, seja a uma parede de distância ou do outro lado do globo. É uma de suas premissas, reduzir fronteiras geradas por fatores de localização geográfica, integrando pessoas de diversas classes, culturas, religiões e hábitos.

Em um recente processo evolutivo, a internet surge envolta de diversos interesses desde a sua criação. Não foi apenas a sua estrutura que sofreu mutações ao longo desse tempo, mas, também, a forma como o ambiente pode ser utilizado. Hoje, aqueles que nascem nessa nova geração, têm crescido com alguns valores e costumes que foram adquiridos a partir de todos os estudos e aperfeiçoamentos do world wide web.

Os jovens contemporâneos foram alfabetizados no mundo conectado. A tecnologia é uma extensão do seu corpo. A utilização dos novos aparatos tecnológicos é aprendida de forma orgânica, à medida que 
é manuseado, dispensando o uso das instruções. Os aplicativos para smartphones são seus aliados, quanto maior o seu poder de comunicação, maior o número de recursos para se expor e revelar suas crenças e ideais, mais sucesso faz com essa faixa da população. A revista Time, de 9 de maio de 2013, ressalta que apesar de egocêntrica e com excesso de autoconfiança, essa nova geração, chamada de Millennials, está apenas se adaptando aos rápidos avanços tecnológicos.

Celulares repletos de aplicativos com acesso a internet são as portas abertas para que essa nova geração entre em contato com o mundo. As inúmeras redes sociais ofertam recursos distintos para chegar a esse objetivo, já que elas são uma alternativa para criar e resgatar uma integração social, eliminando distâncias ao utilizar-se de relacionamentos através do mundo virtual. Recuero (2009, online) afirma que "uma rede social é definida como um conjunto de dois elementos: atores [...] e suas conexões [...]", eles são a razão e o alimento das redes que, segundo Mulgan (1991:21),"[...] são criadas não apenas para comunicar, mas para ganhar posições e melhorar a comunicação", isso quer dizer que, as redes sociais foram criadas com o objetivo de melhorar o processo comunicacional, mas isso só acontece se existirem os usuários para fazer conexões entre si com o objetivo de se comunicar.

Uma rede social torna seu usuário um gerador de conteúdo. De acordo com Zuckerberg (ZUCKERBERG, 2008, apud KIRKPATRICK, 2010), nas redes sociais virtuais existe troca de informações de forma constante, por intermédio de grupos de usuários que se unem com o objetivo de discutir e publicar posts com temas de interesse relevantes ou não.

A rede social que ganhou uma grande visibilidade nos últimos anos foi o Facebook, que foi desenvolvido inicialmente para uso dos fundadores Mark Zuckerberg, Eduardo Saverin, Dustin Moskovitz e Chris Hughes, colegas e estudantes da Universidade de Harvard nos EUA. Lançada em 4 de fevereiro de 2004 para uso de algumas universidades, essa rede social virtual tornou-se aberta no dia 11 de setembro de 2006 e desde então vem conquistando novos usuários em todo o mundo. Abrangendo diversas áreas e segmentos, o Facebook é uma ferramenta que vai além da função de conhecer pessoas.

\begin{abstract}
Quando lançamos o Facebook em 2004, nosso objetivo era criar um jeito mais rico e rápido das pessoas dividirem informação sobre o que estava acontecendo ao seu redor. Pensávamos que dando para as pessoas melhores ferramentas para elas se comunicarem, isso ajudaria a entenderem melhor o mundo, o que faria com que elas tivessem mais poder de transformá-lo (ZUCKERBERG, 2008, apud KIRKPATRICK, 2010).
\end{abstract}

Segundo a matéria "A origem do Facebook" publicada no site do jornal "O Globo" (2012), o Facebook estabeleceu uma ordem na internet, trazendo uma lógica para esse território que não possui fronteiras físicas. De acordo com a citação de Kirsten Bischoff, colaboradora da "Forbes", para a matéria do "O Globo":

\footnotetext{
[...] a visão de Zuckerberg de um "passaporte Facebook" é uma grande sacada, uma estratégia quase premonitória [...]. Ele criou a infraestrutura real para usuários na internet, o que é diferente de uma infraestrutura de rotas comerciais, assemelhando-se mais a um sistema de estradas. A missão do Facebook é servir como mapa segundo o qual o indivíduo pode se mover pela rede e descobrir novas coisas com base nas outras pessoas que vai encontrando, nas pessoas que já conhece e nas recomendações que essas pessoas the dão.
}

Bischoff apresenta essa rede social como uma teia informacional, alimentada pelos usuários, onde se é guiado por suas relações. A quantidade de rotas é proporcional ao número de amigos adicionados, quanto maior o número de conexões, maior a quantidade de informação publicada e maior a probabilidade de entrar em contato com conteúdos novos.

Segundo o site UOL (2013), o Facebook é a maior rede social virtual do mundo, com números estratosféricos de usuários. Se o número representasse a po- 
pulação de um país, seria o terceiro maior do mundo (só perderia para China e Índia). Esse número de pessoas é equivalente ao triplo da população dos EUA. 0 Brasil é o país que mais teve crescimento absoluto de usuários no ano de 2012. Nos seis primeiros meses do ano, 16 milhões de pessoas aderiram ao Facebook. 0 Brasil é o quarto país em número de usuários: com 54 milhões de cadastrados no país, um a cada quatro brasileiros têm uma conta nessa rede social.

O Facebook é uma extensão do mundo, nele é possível ter acesso a cultura de vários países através da parcela do mundo que aderiu a essa rede social. As possibilidades interativas são diversas e permitem variadas formas de contato e geração de informação, facilitado pela adaptação aos gadgets modernos e pela integração com várias outras redes sociais como o Instagram, Twitter, Pinterest, Youtube e Vimeo. No Facebook o usuário é um peregrino que, a cada passo dado, se depara com um universo de informações e novas experiências e que fornece aos outros uma parcela do seu mundo.

\section{UM GRUPO DA ACADEMIA E SUA EXTENSÃO VIRTUAL}

Comentar, curtir, compartilhar, palavras comuns no vocabulário dos usuários da rede social mais acessada no mundo, o Facebook. Nela está disponível uma infinidade de ferramentas que promovem a interação entre duas ou mais pessoas num ambiente virtual, que vão da criação e participação de eventos e grupos de discussão, abertos ou restritos, perpassando pelas páginas de fãs, até uma simples publicação ou bate-papo num perfil. Com toda essa gama de funções a plataforma fornece os subsídios necessários para criar um universo paralelo onde o tempo e o espaço não interferem na comunicação, oferecendo o mundo na web.

A disciplina Multimídia do curso de Design Gráfico da Universidade Tiradentes fez uso dos recursos disponíveis nessa plataforma com o intuito de oferecer uma nova forma de interação com a disciplina objetivando aumentar o nível de participação e discussão dos conteúdos expostos em sala de aula. Para isso, foi utilizado o recurso grupos fechados do Facebook, um local restrito somente aos alunos da disciplina onde podiam ser postadas informações, incitar debates,

compartilhar documentos e experiências, além de fornecer ao professor da disciplina um acompanhamento do desenvolvimento da turma e envio de feedbacks aos seus alunos.

Nesta experiência com a disciplina Multimídia, o grupo foi utilizado para discutir temas iniciais sobre a interatividade na internet. 0 professor iniciava os questionamentos em sua aula expositiva e os alunos as carregavam para o grupo, gerando respostas, novos questionamentos e indicando links com conteúdo complementar. Com a evolução dessa dinâmica, os estudantes começaram a construir textos mais complexos sobre os assuntos discutidos no grupo, disponibilizá-los no próprio grupo onde eram acompanhados pelo professor que sugeria alterações e fazia considerações sobre o conteúdo. Assim que aprovados os textos, os alunos iniciaram o processo de conversão do conteúdo desenvolvido em vídeos, utilizando os recursos acessíveis. Nesta fase, o grupo passou a ser utilizado para agendar visitas a ilha de edição da universidade e para a orientação necessária à construção dos vídeos. 
O resultado final desta experiência foi a viralização dos vídeos construídos pelos alunos por toda a internet e, consequentemente, no Facebook, tornando o conteúdo desenvolvido acessível a todos aqueles que possuíssem interesse, expandindo a experiência de discussões e trocas de informações para todos os usuários da plataforma. Os vídeos foram upados no Youtube, como podemos ver no canal do aluno Dorivaldo Araújo (http:// www.youtube.com/user/Dorivalllfeature=watch) e, após a avaliação do professor, os estes foram dissipados pela rede por e-mail, chats, fóruns, redes sociais e, em especial, no Facebook.

Este grupo mudou a dinâmica da sala de aula nesta disciplina, tendo em vista que os conteúdos lá disponíveis eram massivamente discutidos em aula, tornando a organização mais próxima da horizontalidade. Isso reduziu a percepção de que o professor é o único ser capaz de fornecer novas informações, de que ele é o mestre da verdade absoluta. Dessa forma, foi dada voz aos alunos para que eles trouxessem o seu ponto de vista e suas pesquisas, criando, assim, uma via de mão dupla do conhecimento, onde aluno e professor aprendem juntos com a troca de suas experiências. Outro ponto importante que deve ser elucidado é a questão do contato aproximado gerado pela interação que acontecia na extensão da aula no Facebook, onde o professor encontrava-se disponível para tirar dúvidas e ajudar aos alunos em suas corridas em busca de informação. Tudo isso, sem que houvesse a necessidade de agendar um horário ou aguardar pela aula da semana seguinte, já que o professor os atendia assim que possível quando logado na rede social, ato que acorria diariamente, mediante a um prévio acordo realizado em sala de aula.

A utilização deste recurso beneficiou a todos. Os alunos mais tímidos conseguiam fazer seus questionamentos e participar ativamente das discussões nos grupos, aqueles mais desatentos conseguiam acompanhar tudo que estava acontecendo pois, o conteúdo estava disponível o tempo todo e poderia ser visto e revisto de acordo com as suas necessidades. Todos os estudantes aplicaram a teoria da disciplina na prática e ainda foram, em parte, geradores do conhecimento adquirido na matéria. 0 professor conseguiu acompanhar de perto o desenvolvimento da turma e de cada indivíduo que a compunha, tendo o ritmo da aula ditado pelo desenvolvimento de seus alunos dentro da sala de aula, reforçando o conteúdo nos debates virtuais e incentivando o desenvolvimento de cada um em suas deficiências e potencialidades.

\section{CONCLUSÃO}

O Facebook foi a plataforma mais acertada para a realização desta experiência por ser uma rede social onde todos os alunos estavam inseridos. Fato este, que influenciou na facilidade de utilização dos recursos disponíveis no grupo, o que, por sua vez, tornou completo o aproveitamento de tudo que foi desenvolvido durante a disciplina.

O sistema de monitoria e de autoaprendizagem assistida promovida pela experiência nesta disciplina impulsionou os alunos, influenciando-os a torna-

rem-se autossuficientes e curiosos. Tal fato contribui para retirá-los da acomodação do sistema regular de ensino, onde as informações são despejadas para os estudantes que se comportam como observadores que só enxergam pela fresta aberta pelo professor. Ao tornar o aluno parceiro na geração do conteúdo, um mundo de possiblidades é aberto durante o processo de pesquisa, o que fornece o desenvolvimento da percepção, interpretação e confronto dos conteúdos a partir de sua própria abertura no véu que cobre o conhecimento. 
Ao concluir a disciplina o aluno tornou-se fonte de pesquisa para o mundo, fixando sua marca através de um vídeo, que resumia o conteúdo apreendido durante os cinco meses de estudo e pesquisa, dispo- nibilizado na internet e viralizado no Facebook. Esse mesmo estudante passou a perceber, a partir desta experiência, que ele é capaz de gerar conhecimento e ser referência em pesquisas posteriores.

\section{REFERÊNCIAS}

A maior rede social do mundo tem números estratosféricos. Disponível em: http://tecnologia.uol.com.br/album/2012/08/03/maior-rede-social-do-mundo-facebook-tem-numeros-estratosfericos-conheca.htm. Acesso em: 22 maio 2013.

BARTLETT, Lindsey. In Defense of Millennials. Disponível em: http://linzitheuncritic.wordpress.com/tag/the-me-me-me-generation-by-joel-stein/. Acesso em: 22 maio 2013.

BURGOS, Pedro. Ficar um ano sem internet é um experimento que não prova muita coisa. Disponível em: http://www.oene.com.br/digital-detox/ . Acesso em: 20 maio 2013.

FOREGGER, Sarak K. Uses and Gratifications of Facebook.com. ProQuest LLC: Michigan, 2008. Disponível em: http://books.google.com.br/booksid=0idbf365YBkC\&printsec=frontcoverv=onepage\&q\&f=false. Acesso em: 2 jun 2013.

KIRKPATRICK, David. The Facebook Effect: The Inside Story of the Company That Is Connecting the World . Simon \& Schster, 2010.

MILLER, Paul. I'm still here: back online after a year without the internet. Disponível em: http://www.theverge.com/2013/5/1/4279674/im-still-here-back-online-after-a-year-without-the-internet. Acesso em: 20 maio 2013.

MULGAN, G.J. Communication and Control. Networks and the New Economics of communication. Nova lorque: Guilford Press, 1991.

RECUERO, R.; ZAGO, G. Em Busca das “Redes que Importam": redes sociais e capital social no Twitter. XVIII Encontro da Compós, MG, junho de 2009. Disponível em: http://www.revistas.univerciencia.org/index.php/libero/article/viewFile/6787/6129.pdf. Acesso em: 22 maio 2013

REEVE, Elspeth. Every Every Every Generation has been the Me Me Me Generation. Disponível em: http:// www.theatlanticwire.com/national/2013/05/me-generation-time/65054/. Acesso em: 22 maio 2013.

SILVA, Alexandre Soares. Viva menos e se conecte mais. Disponível em: http://vip.abril.com.br/cultura/papo-vip/viva-menos-e-se-conecte-mais/. Acesso em: 20 maio 2013. 
TEIXEIRA, Carlos Alberto. A origem do Facebook. Disponível em: http://oglobo.globo.com/tecnologia/a-origem-do-facebook-4934191. Acesso em: 5 jun 2013.

1 Bacharel em Design Gráfico - Unit. Pós-Graduando em Artes Visuais Senac. E-mail: edilbertomarcelino@gmail.com

2 Doutora Multidisciplinar em Cultura e Sociedade - Ufba. Mestre em Letras - Ufba. Professora Plena do Programa de Pós-graduação em Educação da Universidade Tiradentes - Unit. Vice-Líder e pesquisadora do Grupo de Pesquisa em Educação, Tecnologias da Informação e Cibercultura - Unit/CNPq e pesquisadora do Grupo Comunicação, Educação e Sociedade -Unit/CNPq. E-mail:crismporto@gmail.com

3 Professor dos cursos de Design Gráfico, Jornalismo e Publicidade \& Propaganda - UNIT. Consultor de Branding (Gestão de Marcas) e Social Media. Bacharel em Design Gráfico - UNIT.Especialização em Marketing e Propaganda-ESM. E-mail: valmirmizio@msn.com

4 Professor - Unit. Coordenador de Tecnologias Educacionais do Núcleo de Educação a Distancia - NEAD Unit. Mestrando em Educação pela Universidade Tiradentes, Especialista em Gestão de Instituições de Ensino Superior - FTC, graduação em Ciência da Computação - UNIFACS. Membro do Grupo de Pesquisa Educação, Tecnologias da Informação e Cibercultura. E-mail: lucas.vale@gmail.com 